Report 2002-016

Insuring against the shortfall risk associated with real options

Heinz Weisshaupt

ISSN 1389-2355 


\title{
Insuring against the shortfall risk associated with real options
}

\author{
Heinz Weisshaupt ${ }^{\dagger}$
}

22.04 .2002

\begin{abstract}
Abstract: Given two assets $S$ and $\tilde{S}$ with price processes two correlated geometric Brownian motions $S_{\bullet}=\left(S_{t}\right)_{t \geq 0}, \tilde{S}_{\bullet}=\left(S_{t}\right)_{t \geq 0}$ and a bond $B$. Suppose that we can only observe $\tilde{S}_{\bullet}$ and can only trade in $\tilde{S}_{\bullet}$ and $B$ or equivalently buy options on $\tilde{S}_{\bullet}$ at time $T=0$. Suppose further that we want to hedge an arbitrary binary option $H$ depending on $S_{T}$; i.e. $H=1_{\left\{S_{T} \in A\right\}}$. Under these conditions it is shown that the best action we can take to minimize the shortfall probability is to buy a binary option $\tilde{H}$ depending on $\tilde{S}_{T}$. Especially the cases $H=1_{\left\{S_{T}<c\right\}}$ and $H=1_{\left\{S_{r}>c\right\}}$ are considered.
\end{abstract}

Keywords: Minimization of shortfall probabilities, BS-Model, (real)-options, correlated price processes, insurance by optimal selection of options (trading strategies).

AMS Subject Classification: 91B28, 60J65, 62P05, 91B30, 62F03

†EURANDOM, TU/e Eindhoven. This paper was partially written at the Department of Financial and Actuarial Mathematics at the TU-Vienna and at the Department of Statistics and Decision Support Systems at Vienna University 


\section{Introduction}

Suppose that we want to insure ourselves against the loss associated with the occurrence of an unfortunate event $E$. This could for example be the event that the development of some product has to be blown off and the loss results from the development costs which accumulated so far. We suppose that the occurrence of the event can be modelled by the fact that a stochastic process $S_{\text {. }}$ is at time $T$ in a certain set. (The stochastic process might for example be the process of estimated total gain of selling the product minus the estimated development costs. $T$ might be some time point where the management evaluates the development.) We suppose further, that there exists an asset traded on

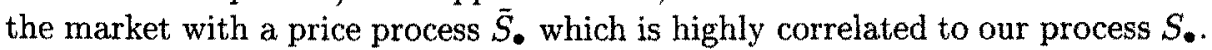
We investigate in this paper how we can insure against the loss associated with the occurrence of the event $E$ by trading in the correlated asset $\tilde{S}_{\bullet}$ and a bond $B$.

This paper is therefore concerned with insurance against risks concerning assets not traded on the market, using options on a correlated asset traded on the market. We consider the important case that the price process of the asset not traded can not be observed between the starting time $t=0$ and the constant stopping time $t=T$. The risk-measure we consider is the shortfall probability, which is in general used to study partial hedging (see the papers [3] and [4] of $\mathrm{H}$. Foellmer and P. Leukert). We will show that under certain conditions (within the Black-Scholes model) the best insurance against such risks is a binary option on the correlated asset. To get a better insight into the economical situation under which such an insurance is desirable we give two examples:

Example 1: Let $\tilde{S}$ denote the stock of a pharmaceutical company and suppose that this company has developed a new drug which we denote by $S$. The market is well informed about this drug and there are only a few clinical trials open. We suppose that there exists a price process $S$. which describes the expected discounted total income the company will get by selling the drug in the future. This process can of course not be observed. The pharmaceutical company now faces the risk that the outstanding clinical trials are negative, which would cause the value $S$. of the drug to decrease dramatically. The price process

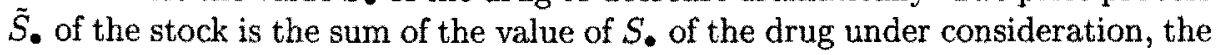
value of all the other products of the pharmaceutical company on the market, and the projected value of new research projects of the company. If we suppose that the market gets only little information about other research projects, but nearly all information about the clinical trials on the new drug during this phase of last clinical trials, there is a high correlation between the process $S$.

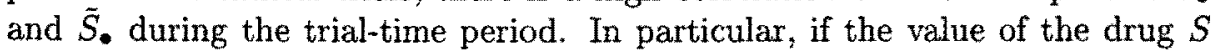
dramatically decreases we know that with high probability also the value of the stock $\tilde{S}$ will dramatically decrease. As already mentioned, the price process $S$. can not be observed. The pharmaceutical company does not try to estimate $S$. 
itself using the outcome of the clinical trials, but it trusts in the opinion of the market which is given by $\tilde{S}_{\text {. }}$. Therefore the pharmaceutical company decides to insure against the risk that the trials are negative (i.e. that the value of $S$ at some time $T$ after the clinical trials becomes smaller than some constant $c$ ) by buying a binary option $H$ on its own stock $\tilde{S}$ (or another stock whose price is assumed to be highly correlated with $S$ ). We will show in this paper, that in the context of the Black-Scholes model this is the best action the company can take in the following sense:

Suppose the company is willing to invest a certain amount of money in options on its own stock to insure itself against the risk that $S_{T}$ falls below a threshold value $c$. If the company wants to maximize the probability that it does not lose more than a prescribed amount of money in case of a negative outcome of the clinical trials, the best action the company can take is to buy a binary option on its own stock $\tilde{S}$.

Example 2: Suppose that a mining company wants to exploit a certain mining area. In order to do this, the mining company has to make some investments. The mineral $M$ which the mining company would produce after this investment is not traded now, but there exists another mineral $\tilde{M}$ with nearly the same compounds and structure which is already traded on the market. We suppose therefore that the price process $S$. of the mineral $M$ is highly correlated with the price process $\tilde{S}$. of the mineral $\tilde{M}$. The mining company faces the following risk: If the price of the mineral $M$ after the period of investment is below the production costs $c$ then the company will make losses. We will show below that a binary option on $\tilde{M}$ is the best action the company can take if it wants to invest money in options on mineral $\bar{M}$ to insure against this risk.

From the viewpoint of real options theory (see the book of M. Amram and N. Kulatilaka (1999) [1]) the investment the companies in example 1 and 2 should make is a real option. A frequently asked question concerning real options is whether we should buy it (make the investment) or not if the price for it is given. However, it is not possible to answer this question from no arbitrage arguments only. Therefore, this article considers instead the question of how we can deal with the risk which arises from buying a real option most efficiently. We will obtain explicit formulas for the relevant binary options in the cases $S_{T}<c$ and $S_{T}>c$ which are most interesting from a practical point of view.

How important real options are in practice can be estimated from the articles of T. A. Luehrman (1998) [6] and of M. Amram and N. Kulatilaka (1999) [2]. In these articles it is clearly pointed out how real options can be used as tools to decrease the risks of management decisions and how investment strategies can be translated into the terminology of real options. The present paper now shows how the risk of investment can be further decreased by insuring the real 
option by some option traded on the financial market, and by this transferring the risk to the market. (Of course in practice the company will have to pay a risk premium for this transfer, which is not considered in our model.)

In this paper we do not use the heavy machinery of stochastic analysis. Instead we make use of a version of the Neyman-Pearson Lemma, some easy lemmas on conditional expectations and the fact that the Black-Scholes Model is complete. By this we circumnavigate all technical difficulties.

We remark finally that in contrast to the paper [3] we do not minimize the shortfall probability of a strategy on a stochastic process with respect to a constraint on its equivalent martingale measure; i.e. we do not minimize the shortfall probability of a strategy based on a process observable at any time with respect to this process. Instead we minimize the shortfall probability of a strategy based on an observable process with respect to another not observable process. The price for this more general situation is that we can only consider binary options while in [3] arbitrary options are considered (and the case of a European call is explicitly calculated in the Black-Scholes Model). Another important difference to the paper [3] is, that our method developed so far only works if the market given by the observable process and the bond is complete while in [3] also incomplete markets are considered.

\section{The Setting}

Let $(\Omega, \mathcal{F}, \mathbb{P})$ be a probability space and let $W$ and $\tilde{W}$ be two standard Brownian motions defined on this space. Suppose that $W$ and $\tilde{W}$ are correlated with fixed correlation $\rho \in(-1,1) \backslash\{0\}$; i.e. there exists a standard Brownian motion $\tilde{W}^{\perp}$ (also defined on $\Omega$ ) such that $\tilde{W}^{\perp}$ is independent of $\tilde{W}$ and

$$
W=\rho \tilde{W}+\sqrt{1-\rho^{2}} \tilde{W}^{\perp} .
$$

Equivalently $\tilde{W}$ can be expressed as

$$
\tilde{W}=\rho W+\sqrt{1-\rho^{2}} W^{\perp}
$$

with $W^{\perp}$ a standard Brownian motion independent of $W$.

We denote by $\left(\tilde{\mathcal{F}}_{t}\right)_{t \in[0, T]}$ the natural (right continuous, saturated) filtration generated by $\tilde{W}$ up to time $T$. We assume that $W_{0}=\tilde{W}_{0}=0$ so that $\mathcal{F}_{0}$ becomes trivial.

Let $S, \tilde{S}$ be two assets ( $S$ not traded and $\tilde{S}$ traded). We denote by $S_{\bullet}$ and $\tilde{S}$. the price processes of $S$ and $\tilde{S}$ and by $S_{t}$ and $\tilde{S}_{t}$ these price processes at time 
$t$. Let $B$ denote the bond. For simplicity of notation we assume in the following that the interest rate is equal to 0 and that the price processes are given by

$$
\begin{gathered}
\frac{d S_{t}}{S_{t}}=d W_{t}, S_{0}=1 \text { and } \\
\frac{d \tilde{S}_{t}}{\tilde{S}_{t}}=d \tilde{W}_{t}, \tilde{S}_{0}=1 .
\end{gathered}
$$

More general situations are considered in the appendix. As can be easily verified by Itô's formula (see the book of Steele 2001 [7] Chapter 8) solutions of these equations are given by

$$
S_{t}=e^{W_{t}-\frac{t}{2}} \text { and } \tilde{S}_{t}=e^{\bar{W}_{t}-\frac{t}{2}} .
$$

These solutions are also unique (see [7] Chapter 9.4).

Thus we obtain that the joint distribution of $\ln \left(S_{T}\right)+\frac{T}{2}$ and $\ln \left(\tilde{S}_{T}\right)+\frac{T}{2}$ is multivariate normal and given by

$$
\left(\begin{array}{l}
\ln \left(S_{T}\right)+\frac{T}{2} \\
\ln \left(\tilde{S}_{T}\right)+\frac{T}{2}
\end{array}\right) \sim N\left(\left(\begin{array}{l}
0 \\
0
\end{array}\right)\left(\begin{array}{cc}
T & \rho T \\
\rho T & T
\end{array}\right)\right) .
$$

From (2) the conditional distribution of $\ln \left(\tilde{S}_{T}\right)+\frac{T}{2}$ under the condition that $\ln \left(S_{T}\right)+\frac{T}{2}=a$ can be inferred. According to Exercise 3.3 of [7] we obtain that

$$
\mathbb{P}\left[\ln \left(\tilde{S}_{T}\right)+\frac{T}{2} \in \cdot \ln \left(S_{T}\right)+\frac{T}{2}=a\right] \sim N\left(a \rho, T\left(1-\rho^{2}\right)\right) .
$$

We are interested in the following situation:

Suppose we would like to hedge a binary option on $S$; i.e. we would like to hedge $H=1_{A_{T}}\left(S_{T}\right)$ (with $A_{T}$ a Borel set in $[0, \infty)$ ), but at time $t \in[0, T]$ we are only given the information $\left(\tilde{S}_{t^{\prime}}\right)_{t^{\prime} \in[0, t]}$ and we are only allowed to invest in the bond $B$ and in the stock $\tilde{S}$. So we are interested in the trading strategies $\phi(t)=$ $\left(\phi_{B}(t), \phi_{\bar{S}}(t)\right)$, where $\phi_{B}(t)$ denotes the amount of money held in the bond and $\phi_{\tilde{S}}(t)$ denotes the number of stocks held at time $t$. We assume that $\phi(t)$ is a predictable process with respect to the filtration $\left(\tilde{\mathcal{F}}_{t}\right)$, that it is self-financing and at any time $t \in[0, T]$ of non negative value $V_{T}^{\phi}:=\phi_{\bar{S}}(t) \tilde{S}_{t}+\phi_{B}(t)$. We further suppose that the initial wealth is given by some fixed value $v_{0}=V_{0}^{\phi} \in[0,1]$. We call such strategies admissible and denote the set of all admissible strategies by $\Phi$. We note that a self financing strategy fulfills the following stochastic differential equation:

$$
d\left(\phi_{\bar{S}}(t) \tilde{S}_{t}\right)+d \phi_{B}(t)=\phi_{\bar{S}}(t) d \tilde{S}_{t}, \quad \phi_{\bar{S}}(0) \tilde{S}_{0}+\phi_{B}(0)=v_{0}
$$


We introduce further the following notations:

We denote by $\mathbb{R}$ the real line and let $\mathbb{R}_{+}=[0, \infty)$. By $\mathcal{B}$ respectively $\mathcal{B}_{+}$we denote the family of Borel sets on $\mathbb{R}$ respectively $\mathbb{R}_{+}$. We let $p r_{s}: \mathbb{R}^{[0, T]} \mapsto \mathbb{R}$ be the mapping which projects $\mathbb{R}^{[0, T]}$ onto its $s$-th coordinate; i.e. $p r_{s}\left(\left(x_{t}\right)_{t \in[0, T]}\right)=$ $x_{s}$. We denote by $\mathcal{B}_{+}^{[0, T]}$ the $\sigma$-algebra of subsets of $\mathbb{R}_{+}^{[0, T]}$ generated by $\bigcup_{s \in[0, T]} p r_{s}^{-1}\left(\mathcal{B}_{+}\right)$.

\section{The Problem and the Main Theorems}

We consider a set $A_{T} \in \mathcal{B}_{+}$, the binary option $H:=1_{A_{T}}\left(S_{T}\right)$, and the initial value $V_{0}^{\phi}:=v_{0} \in[0,1]$. We would like to solve the following problem; What are the strategies $\phi$ such that the shortfall probability i.e. the probability that the value $V_{T}^{\phi}$ is strictly smaller than $H$ is minimized within the class of strategies $\Phi ?$

To be more precise: Find a strategy $\phi$ which is optimal with respect to the criterion:

$$
\left.\begin{array}{c}
\min \left\{\mathbb{P}\left(V_{T}^{\phi}<1_{A_{T}}\left(S_{T}\right)\right): \phi \in \Phi\right\} \\
\text { subject to the constraint } V_{0}^{\phi}=v_{0} .
\end{array}\right\}
$$

We will prove the following theorems:

Theorem 1: Given $T>0, A_{T} \in \mathcal{B}_{+}$, and $v_{0} \in[0,1]$, there exists a set $C_{T} \in \mathcal{B}_{+}$and an admissible strategy $\phi=\left(\phi_{B}, \phi_{\tilde{S}}\right)$ which completely replicates $1_{C_{T}}\left(\tilde{S}_{T}\right)$ and solves (4).

In some special cases it is possible to obtain the solution of problem (4) in a more specific form:

Theorem 2: Let $F_{T}$ be the distribution function of $N(0, T)$, let $\bar{c}=e^{F_{T}^{-1}\left(v_{0}\right)-\frac{T}{2}}$ and $c=e^{F_{T}^{-1}\left(1-v_{0}\right)-\frac{T}{2}}$. Then the following relations hold:

1. $\left\{\begin{array}{l}\text { If } \rho>0 \text { and } A_{T} \text { is of the form }(0, a], \text { then the set } C_{T} \text { in Theorem } \\ 1 \text { is given by } C_{T}=(0, \bar{c}] \text { a.s. }\end{array}\right.$

2. $\left\{\right.$ If $\rho>0$ and $A_{T}$ is of the form $[a,+\infty)$, then the set $C_{T}$ in Theorem 1 is given by $C_{T}=[\underline{c}, \infty)$ a.s.

3. $\left\{\begin{array}{l}\text { If } \rho>0 \text { and } A_{T} \text { is bounded and bounded away from } 0, \text { then the } \\ \text { set } C_{T} \text { in Theorem } 1 \text { is a.s. bounded and a.s. bounded away from } \\ 0 .\end{array}\right.$ 
4. $\left\{\begin{array}{l}\text { If } \rho<0 \text { and } A_{T} \text { is of the form }(0, a] \text {, then the set } C_{T} \text { in Theorem } \\ 1 \text { is given by } C_{T}=\left[\frac{1}{\underline{e}} e^{-T}, \infty\right) \text { a.s. }\end{array}\right.$

5. $\left\{\begin{array}{l}\text { If } \rho<0 \text { and } A_{T} \text { is of the form }[a,+\infty) \text {, then the set } C_{T} \text { in } \\ \text { Theorem } 1 \text { is given by } C_{T}=\left(0, \frac{1}{\bar{c}} e^{-T}\right] \text { a.s. }\end{array}\right.$

6. $\left\{\begin{array}{l}\text { If } \rho<0 \text { and } A_{T} \text { is bounded and bounded away from } 0, \text { then the } \\ \text { set } C_{T} \text { in Theorem } 1 \text { is a.s. bounded and a.s. bounded away from } \\ 0 .\end{array}\right.$

Notice that $\bar{c}$ and $\underline{c}$ are independent of $a$ and $|\rho|$.

Theorem 3: Let $\varepsilon, a, T>0$ be fixed. Let $\rho$ be the correlation of $W$ and $\tilde{W}$, let $A_{T}=(0, a]$, and let $\phi$ be the solution (granted by Theorem 1) of (4) for $v_{0}=\mathbb{E}_{\not P}\left(1_{(0, a]}\left(S_{T}\right)\right)+\varepsilon$. Then the shortfall probability $\operatorname{Err}(\rho, \varepsilon, a, T)$ defined by $\operatorname{Err}(\rho, \varepsilon, a, T):=\mathbb{P}\left(V_{T}^{\phi}<1_{(0, a)}\left(S_{T}\right)\right)$, tends for $\rho \rightarrow 1$ quicker to 0 than any polynomial in $(1-\rho)$.

Since $V_{T}^{\phi} \geq 0$ by the admissibility of $\phi$ and because of the special structure of $H$ the constraint optimization problem (4) is equivalent to:

$$
\left.\begin{array}{c}
\min \left\{\mathbb{P}\left(V_{T}^{\phi}<1 \mid 1_{A_{T}}\left(S_{T}\right)=1\right): \phi \in \Phi\right\} \\
\text { subject to the constraint } V_{0}^{\phi}=v_{0}
\end{array}\right\}
$$

which is further equivalent with

$$
\left.\begin{array}{c}
\max \left\{\mathbb{P}\left(V_{T}^{\phi} \geq 1 \mid S_{T} \in A_{T}\right): \phi \in \Phi\right\} \\
\text { subject to the constraint } V_{0}^{\phi}=v_{0}
\end{array}\right\}
$$

We denote the wealth at time $t$ along a path $\omega$ under the strategy $\phi$ by $V_{i}^{\phi}(\omega)$. We let $C_{\phi} \in \mathbb{R}^{[0, T]}$ be the set of all paths $\omega$ of $\tilde{S}$. for which $V_{T}^{\phi}(\omega) \geq 1$; i.e.

$$
C_{\phi}=\left\{\omega \in \mathbb{R}^{[0, T]}: V_{T}^{\phi}(\omega) \geq 1\right\} .
$$

Notice that for all $\phi$ which solve (5) we have

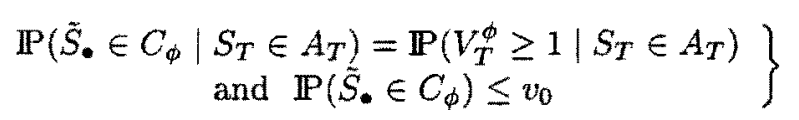

We try to solve the more general optimization problem (recall that we assumed $v_{0} \in[0,1]$ ) 


$$
\left.\begin{array}{c}
\max \left\{\mathbb{P}\left(\tilde{S}_{\bullet} \in C \mid S_{T} \in A_{T}\right): C \in \mathcal{B}_{+}^{[0, T]}\right\} \\
\text { subject to the constraint } \mathbb{P}\left(\tilde{S}_{\bullet} \in C\right)=v_{0} .
\end{array}\right\}
$$

If we can find a set $C$ which solves (7) and for which there exists a $\phi$ such that $C=C_{\phi}$ this $\phi$ fulfills (6). By (6) and the fact that it fulfills $\mathbb{P}\left(\tilde{S}_{\bullet} \in C\right)=v_{0}$ this $\phi$ is also a solution of the optimization problems (5) and (4). We will show in this paper that it is indeed possible to find such a set $C$ and a $\phi$ with $C=C_{\phi}$ and thus obtain a solution of (5) and (4).

Problem (7) is now (partially) solved by an application of the following Proposition, which can be viewed as a special version of the Neyman-Pearson Lemma.

Proposition 1: Let $Q$ and $R$ be measures on a measurable space $(\hat{\Omega}, \mathcal{D})$. Let $R$ be nonatomic and $Q$ absolutely continuous with respect to $R$. Then for any $v_{0} \in(0,1)$ there exists a unique $\beta \in[0, \infty)$ such that the family $\mathcal{C}$ of all sets $C \in \mathcal{D}$ which solve

$$
\max \{Q(C): C \in \mathcal{D}\} \text { subject to the constraint } R(C)=v_{0}
$$

is nonempty and equals the family of all sets $C \in \mathcal{D}$ which fulfill

$$
\left.\begin{array}{c}
\left\{\omega \in \Omega: \frac{d Q}{d R}(\omega)>\beta\right\} \subseteq C \subseteq\left\{\omega \in \Omega: \frac{d Q}{d R}(\omega) \geq \beta\right\} \\
\text { and } R(C)=v_{0} .
\end{array}\right\}
$$

Proof: This Proposition can be be proved in an analogous way as the Neyman-Pearson Lemma. For a proof of this Lemma see for example the book of E.L. Lehmann [5].

We define probability measures $Q^{A_{T}}$ and $R$ on $\left(\mathbb{R}_{+}^{[0, T]}, \mathcal{B}_{+}^{[0, T]}\right)$ by

$$
Q^{A_{T}}(D):=\mathbb{P}\left(\tilde{S}_{\bullet} \in D \mid S_{T} \in A_{T}\right) \text { and } R(D):=\mathbb{P}\left(\tilde{S}_{\bullet} \in D\right)
$$

for $D \in \mathcal{B}_{+}^{[0, T]}$. We will show below in the proof of Theorem 1 that $R$ and $Q^{A_{T}}$ are nonatomic and that $Q^{A_{T}}$ is absolutely continuous with respect to $R$.

If we let $\Omega=\mathbb{R}_{+}^{[0, T]}, \mathcal{D}=\mathcal{B}_{+}^{[0, T]}, Q=Q^{A_{T}}$, then by definition (10) the optimization problems (8) and (7) become identical and thus by an application of Proposition 1, reformulating (9) in terms of (10) we get that there exists a unique $\beta \in \mathbb{R}_{+}$such that a set $C \in \mathcal{B}_{+}^{[0, T]}$ is a solution of (7) if and only if 


$$
\begin{gathered}
C_{>\beta}:=\left\{\left(x_{t}\right)_{t \in[0, T]} \in \mathbb{R}_{+}^{[0, T]}: \frac{d Q^{A T}}{d R}\left(\left(x_{t}\right)_{t \in[0, T]}\right)>\beta\right\} \subseteq C \\
C \subseteq\left\{\left(x_{t}\right)_{t \in[0, T]} \in \mathbb{R}_{+}^{[0, T]}: \frac{d Q^{A_{T}}}{d R}\left(\left(x_{t}\right)_{t \in[0, T]}\right) \geq \beta\right\}=: C_{\geq \beta} \\
\text { and } R(C)=v_{0}
\end{gathered}
$$

So to solve the problem (7) we only have to calculate $\frac{d Q^{A} T}{d R}$ and $\beta$ and then choose $C$ according to condition (11).

In the next section we show that there exists a solution $C$ of our optimization problem (7) which is of the form $C=\left\{\left(x_{t}\right)_{t \in[0, T]}: x_{T} \in C_{T}\right\}=p r_{T}^{-1}\left(C_{T}\right)$ for some set $C_{T} \in \mathcal{B}_{+}$.

\section{The proof of Theorem 1}

For the proof we need some Lemmata:

Lemma 1: Let $Q$ and $R$ be probability measures on $(\hat{\Omega}, \mathcal{D})$ and let $\mathcal{D}_{T} \subseteq \mathcal{D}$ be a sub $\sigma$-algebra. Suppose that

$$
\forall D \in \mathcal{D}, \quad \mathbb{E}_{Q}\left(1_{D} \mid \mathcal{D}_{T}\right)=\mathbb{E}_{R}\left(1_{D} \mid \mathcal{D}_{T}\right) \text { a.s. }
$$

and that $\left.Q\right|_{\mathcal{D}_{T}}$ is absolutely continuous with respect to $\left.R\right|_{\mathcal{D}_{T}}$. Then $Q$ is absolutely continuous with respect to $R$ and

$$
\frac{d Q}{d R}=\frac{\left.d Q\right|_{\mathcal{D}_{T}}}{\left.d R\right|_{\mathcal{D}_{T}}}
$$

Proof: We have to show that for all $D \in \mathcal{D}$

$$
Q(D)=\int_{D} \frac{d Q \mid \mathcal{D}_{T}}{d R \mid \mathcal{D}_{T}} d R
$$

and do this by the following calculation:

$$
\begin{aligned}
& Q(D)=\mathbb{E}_{Q}\left(1_{D}\right)=\mathbb{E}_{\left.Q\right|_{\mathcal{D}_{T}}}\left(\mathbb{E}_{Q}\left(1_{D} \mid \mathcal{D}_{T}\right)\right)=\mathbb{E}_{\left.Q\right|_{D_{T}}}\left(\mathbb{E}_{R}\left(1_{D} \mid \mathcal{D}_{T}\right)\right) \\
& =\left.\int \mathbb{E}_{R}\left(1_{D} \mid \mathcal{D}_{T}\right) d Q\right|_{\mathcal{D}_{T}}=\left.\int \mathbb{E}_{R}\left(1_{D} \mid \mathcal{D}_{T}\right) \frac{\left.d Q\right|_{\mathcal{D}_{T}}}{\left.d R\right|_{\mathcal{D}_{T}}} d R\right|_{\mathcal{D}_{T}} \\
& =\mathbb{E}_{\left.R\right|_{\mathcal{D}_{T}}}\left(\frac{\left.d Q\right|_{\mathcal{D}_{T}}}{d R \mid \mathcal{D}_{T}} \mathbb{E}_{R}\left(1_{D} \mid \mathcal{D}_{T}\right)\right)=\mathbb{E}_{\left.R\right|_{\mathcal{D}_{T}}}\left(\mathbb{E}_{R}\left(\frac{d Q \mid \mathcal{D}_{T}}{d R \mid \mathcal{D}_{T}} 1_{D} \mid \mathcal{D}_{T}\right)\right) \\
& =\mathbb{E}_{R}\left(\frac{d Q \mid \mathcal{D}_{T}}{\left.d R\right|_{\mathcal{D}_{T}}} 1_{D}\right)=\int_{D} \frac{\left.d Q\right|_{\mathcal{D}_{T}}}{\left.d R\right|_{\mathcal{D}_{T}}} d R
\end{aligned}
$$


Here the $3^{\text {rd }}$ equality follows from the hypothesis and the $5^{\text {th }}$ and $7^{\text {th }}$ equalty follow since $\frac{\left.d Q\right|_{\mathcal{D}_{T}}}{\left.d R\right|_{\mathcal{D}_{T}}}$ is $\mathcal{D}_{T}$ measurable.

Next we define for $A_{T} \in \mathcal{B}_{+}$the measures $Q_{T}^{A_{T}}$ and $R_{T}$ on $\left(\mathbb{R}_{+}, \mathcal{B}_{+}\right)$by

$$
\left.\begin{array}{r}
Q_{T}^{A_{T}}\left(D_{T}\right):=\mathbb{P}\left(\tilde{S}_{T} \in D_{T} \mid S_{T} \in A_{T}\right) \text { and } \\
R_{T}\left(D_{T}\right):=\mathbb{P}\left(\tilde{S}_{T} \in D_{T}\right) \text { with } D_{T} \in \mathcal{B}_{+} .
\end{array}\right\}
$$

For ease of notation and calculations we will also consider the measures $\hat{Q}_{T}^{A_{T}}$ and $\hat{R}_{T}$ which are defined on $(\mathbb{R}, \mathcal{B})$ by

$$
\hat{Q}_{T}^{A_{T}}\left(D_{T}\right):=Q_{T}^{A_{T}}\left(\left\{x: \ln (x)+\frac{T}{2} \in D_{T}\right\}\right)
$$

and

$$
\hat{R}_{T}\left(D_{T}\right):=R_{T}\left(\left\{x: \ln (x)+\frac{T}{2} \in D_{T}\right\}\right) .
$$

If we let $\ln \left(A_{T}\right)+\frac{T}{2}:=\left\{\ln (x)+\frac{T}{2}: x \in A_{T}\right\}$ we obtain

$$
\left.\begin{array}{c}
\hat{Q}_{T}^{A T}\left(D_{T}\right)=\mathbb{P}\left(\ln \left(\tilde{S}_{T}\right)+\frac{T}{2} \in D_{T} \mid \ln \left(S_{T}\right)+\frac{T}{2} \in \ln \left(A_{T}\right)+\frac{T}{2}\right), \\
\hat{R}_{T}\left(D_{T}\right)=\mathbb{P}\left(\ln \left(\hat{S}_{T}\right)+\frac{T}{2} \in D_{T}\right) \text { for } D_{T} \in \mathcal{B} .
\end{array}\right\}
$$

Since by (2) the random variable $\ln \left(S_{T}\right)+\frac{T}{2}$ is $\mathrm{N}(0, \mathrm{~T})$ distributed and by (3) the conditional distribution of $\ln \left(\tilde{S}_{T}\right)+\frac{T}{2}$ under the condition that $\ln \left(S_{T}\right)+\frac{T}{2}=$ $a$ is $N\left(a \rho, T\left(1-\rho^{2}\right)\right)$ it is easy to obtain from (13) that the measures $\hat{Q}_{T}^{A T}$ and $\hat{R}_{T}$ are absolutely continuous with respect to Lebesgue measure $\lambda$ on $(\mathbb{R}, \mathcal{B})$ and their densities are given by

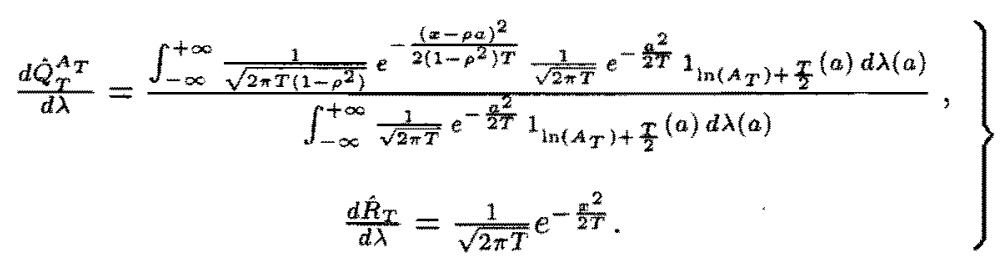

Thus $\hat{Q}_{T}^{A_{T}}$ is absolutely continuous with respect to $\hat{R}_{T}$ and the RadonNykodym derivative is given by:

$$
\frac{d\left(\hat{Q}_{T}^{A_{T}}\right)}{d\left(\hat{R}_{T}\right)}(x)=\frac{\int_{-\infty}^{+\infty} e^{-\frac{(x-\rho \alpha)^{2}}{2\left(1-\rho^{2}\right) T}} e^{-\frac{a^{2}}{2 T}} 1_{\ln \left(A_{T}\right)+\frac{T}{2}(a) d \lambda(a)}}{\sqrt{1-\rho^{2}} e^{-\frac{x^{2}}{2 T}} \int_{-\infty}^{+\infty} e^{-\frac{a^{2}}{2 T}} 1_{\ln \left(A_{T}\right)+\frac{T}{2}}(a) d \lambda(a)}
$$

From this we get immediately: 
Lemma 2: The measure $R_{T}$ is nonatomic and the measure $Q_{T}^{A_{T}}$ is absolutely continuous with respect to $R_{T}$. The Radon-Nykodym derivative is given by

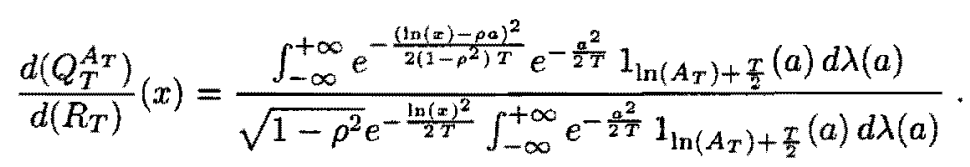

Lemma 3: Let $Q^{A_{T}}$ and $R$ be given by (10) and let $\mathcal{B}_{T} \subset \mathcal{B}_{+}^{[0, T]}$ be the $\sigma$-algebra generated by the projection ${ }^{2} T$; i.e. let

$$
D \in \mathcal{B}_{T} \Leftrightarrow D=p_{T}^{-1}\left(D_{T}\right) \text { for some set } D_{T} \in \mathcal{B}_{+} .
$$

Then we have for all $D \in \mathcal{D}$

$$
\mathbb{E}_{Q^{A_{T}}}\left(1_{D} \mid \mathcal{B}_{T}\right)=\mathbb{E}_{R}\left(1_{D} \mid \mathcal{B}_{T}\right) \text { a.s. }
$$

Proof: All equalities in this proof are to be considered in the almost sure sense.

$$
\begin{aligned}
& Q^{A_{T}}\left(D \mid \mathcal{B}_{T}\right)\left(\left(x_{t}\right)_{t \in[0, T]}\right)=\mathbb{P}\left(\tilde{S}_{\bullet} \in D \mid S_{T} \in A_{T}, \tilde{S}_{T}=x_{T}\right) \\
& =\mathbb{P}\left(\begin{array}{l}
\ln \left(\tilde{S}_{\bullet}\right)+\frac{T}{2} \in \ln (D)+\frac{T}{2} \\
\ln \left(S_{T}\right)+\frac{T}{2} \in \ln \left(A_{T}\right)+\frac{T}{2}, \ln \left(\tilde{S}_{T}\right)+\frac{T}{2}=\ln \left(x_{T}\right)+\frac{T}{2}
\end{array}\right) \\
& =\mathbb{P}\left(\tilde{W} \in \ln (D)+\frac{T}{2} \mid W(T) \in \ln \left(A_{T}\right)+\frac{T}{2}, \tilde{W}(T)=\ln \left(x_{T}\right)+\frac{T}{2}\right) \\
& =\mathbb{P}\left(\begin{array}{l}
\tilde{W} \in \ln (D)+\frac{T}{2} \mid \\
\rho \tilde{W}(T)+\sqrt{1-\rho^{2}} \tilde{W}^{\perp} \in \ln \left(A_{T}\right)+\frac{T}{2}, \tilde{W}(T)=\ln \left(x_{T}\right)+\frac{T}{2}
\end{array}\right) \\
& =\mathbb{P}\left(\begin{array}{l}
\tilde{W} \in \ln (D)+\frac{T}{2} \mid \\
\rho\left[\ln \left(x_{T}\right)+\frac{T}{2}\right]+\sqrt{1-\rho^{2}} \tilde{W}^{\perp} \in \ln \left(A_{T}\right)+\frac{T}{2}, \tilde{W}(T)=\ln \left(x_{T}\right)+\frac{T}{2}
\end{array}\right) \\
& =\mathbb{P}\left(\begin{array}{l}
\tilde{W} \in \ln (D)+\frac{T}{2} \mid \\
\tilde{W}^{\perp} \in \frac{\ln \left(A_{T}\right)+\frac{T}{2}-\rho\left(\ln \left(x_{T}\right)+\frac{T}{2}\right)}{\sqrt{1-\rho^{2}}}, \tilde{W}(T)=\ln \left(x_{T}\right)+\frac{T}{2}
\end{array}\right) \\
& =\mathbb{P}\left(\tilde{W} \in \ln (D)+\frac{T}{2} \mid \tilde{W}(T)=\ln \left(x_{T}\right)+\frac{T}{2}\right) \\
& =\mathbb{P}\left(\ln \left(\tilde{S}_{\bullet}\right) \in \ln (D)+\frac{T}{2} \mid \ln \left(\tilde{S}_{T}\right)+\frac{T}{2}=\ln \left(x_{T}\right)+\frac{T}{2}\right) \\
& =\mathbb{P}\left(\tilde{S}_{\bullet} \in D \mid \tilde{S}_{T}=x_{T}\right)=R\left(\tilde{S}_{\bullet} \in D \mid \mathcal{B}_{T}\right)\left(\left(x_{t}\right)_{t \in[0, T]}\right) .
\end{aligned}
$$

Here the $7^{\text {th }}$ equality holds since $\tilde{W}$ and $\tilde{W}^{\perp}$ are independent. $\square$ 
Remark: The measures $Q_{T}^{A_{T}}$ and $R_{T}$ are related to $Q^{A_{T}}$ and $R$ by

$$
Q_{T}^{A_{T}}\left(D_{T}\right)=\left.Q^{A_{T}}\right|_{\mathcal{B}_{T}}\left(p r_{T}^{-1}\left(D_{T}\right)\right) \text { and } R_{T}\left(D_{T}\right)=\left.R\right|_{\mathcal{B}_{T}}\left(p r_{T}^{-1}\left(D_{T}\right)\right)
$$

for all $D_{T} \in \mathcal{B}_{T}$. Therefore we get by (16) and Lemma 2 that $\left.d Q^{A_{T}}\right|_{\mathcal{B}_{T}}$ is absolutely continuous with respect to $\left.d R\right|_{\mathcal{B}_{T}}$ and thus by (16)

$$
\frac{\left.d Q^{A_{T}}\right|_{\mathcal{B}_{T}}}{\left.d R\right|_{\mathcal{B}_{T}}}\left(\left(x_{t}\right)_{t \in[0, T]}\right)=\frac{d Q_{T}^{A_{T}}}{d R_{T}}\left(x_{T}\right) \text { a.s. . }
$$

An application of Lemma 1 and Lemma 3 with $\hat{\Omega}=\mathbb{R}_{+}^{[0, T]}, \mathcal{D}=\mathcal{B}_{+}, \mathcal{D}_{T}=\mathcal{B}_{T}$ and $Q=Q^{A_{T}}$ gives that

$$
\frac{d Q^{A_{T}}}{d R} \text { exists and } \frac{d Q^{A_{T}}}{d R}\left(\left(x_{t}\right)_{t \in[0, T]}\right)=\frac{\left.d Q^{A_{T}}\right|_{\mathcal{B}_{T}}}{\left.d R\right|_{\mathcal{B}_{T}}}\left(\left(x_{t}\right)_{t \in[0, T]}\right) \text { a.s. }
$$

which together with (17) gives

$$
\frac{d Q^{A_{T}}}{d R}\left(\left(x_{t}\right)_{t \in[0, T]}\right)=\frac{d Q_{T}^{A_{T}}}{d R_{T}}\left(x_{T}\right) \text { a.s. }
$$

We thus see that there exists a version of $\frac{d Q^{A} T}{d R}\left(\left(x_{t}\right)_{t \in[0, T]}\right)$ which only depends on the last coordinate $x_{T}$ of $\left(x_{t}\right)_{t \in[0, T]}$. If we use this version in the definition of $C_{>\beta}$ and $C_{\geq \beta}$, then whether a point $\left(x_{t}\right)_{t \in[0, T]}$ is in the set $C_{>\beta}$ or $C_{\geq \beta}$ defined in (11), depends only on its last coordinate; i.e. it is possible to choose $C_{>\beta}$ and $C_{\geq \beta}$ such that we have

$$
\left.\begin{array}{c}
C_{>\beta}=p r_{T}^{-1}\left(p r_{T}\left(C_{>\beta}\right)\right) \text { and } C_{\geq \beta}=p r_{T}^{-1}\left(p r_{T}\left(C_{\geq \beta}\right)\right) ; \\
\text { i.e. } C_{>\beta}, C_{\geq \beta} \in \mathcal{B}_{T} .
\end{array}\right\}
$$

Since $R_{T}$ is nonatomic by Lemma 2 and $R_{T}\left(p r_{T}(D)\right)=\left.R\right|_{\mathcal{B}_{T}}(D)$ for all $D \in \mathcal{B}_{T}$ we see that also $\left.R\right|_{\mathcal{B}_{T}}$ is nonatomic. that

By (19) the sets $C_{>\beta}$ and $C_{\geq \beta}$ are $\mathcal{B}_{T}$-measurable. We conclude from (11)

$$
\left.R\right|_{\mathcal{B}_{T}}\left(C_{>\beta}\right) \leq v_{0} \leq\left. R\right|_{\mathcal{B}_{T}}\left(C_{\geq \beta}\right) .
$$

Since $\left.R\right|_{\mathcal{B}_{T}}$ is nonatomic we conclude from (20) that there exists a $\mathcal{B}_{T^{-}}$ measurable set $C$ such that $C_{>\beta} \subseteq C \subseteq C_{\geq \beta}$ and $\left.R\right|_{\mathcal{B}_{T}}(C)=v_{0}$. Since $\mathcal{B}_{T}$-measurable sets are of the form $C=\left\{\left(\left(x_{t}\right)_{t \in[0, T]}\right): x_{T} \in C_{T}\right\}$ we see that there exists a solution of (11) and thus of $(7)$ of the desired form.

We thus obtain the following Lemma: 
Lemma 4: The measure $R$ defined by (10) is nonatomic and the measure $Q^{A}$ defined by (10) is absolutely continuous with respect to $R$. The RadonNykodym derivative $\frac{d Q^{A} T}{d R}: \mathbb{R}^{[0, T]} \mapsto \mathbb{R}$ can be chosen to depend only on the last coordinate and is given with respect to this coordinate by Lemma 2. The sets $C_{>\beta}$ and $C_{\geq \beta}$ can be chosen to depend also only on their last coordinate; i.e. they can be chosen to be elements of $\mathcal{B}_{T}^{+}$. There exists a set $C \in \mathcal{B}_{T}$ which solves (7); i.e. there exists a solution $C \in \mathbb{I}^{[0, T]}$ of (7) which depends only on the last coordinate. There exists a trading strategy $\phi$ which replicates $1_{C}\left(\tilde{S_{\bullet}}\right)$ and this strategy solves (4).

Proof: That $R$ is nonatomic follows from Lemma 2 and (16). That $Q^{A_{T}}$ is absolutely continuous with respect to $R$ is a consequence of Lemma 2 and (18). By (18) we also know, that $\frac{d Q^{A} T}{d R}: \mathbb{R}^{[0, T]} \mapsto \mathbb{R}$ can be chosen to depend only on its last coordinate and that again by $(18)$ it is given with respect to this coordinate by Lemma 2 . That the sets $C_{>\beta}$ and $C_{\geq \beta}$ can be chosen to depend only on their last coordinate is the assertion of (19), and that there exists a solution $C$ of (7) which depends only on its last coordinate is the assertion of the paragraph preceding the Lemma, which is based on $(20)$. Thus $1_{C}$ is a binary option on $\tilde{S}$ and by completeness of the Black-Scholes model there exists a trading strategy $\phi$ which completely replicates $1_{C}\left(\tilde{S}_{\bullet}\right)$, if the initial wealth $v_{0}$ equals $\mathbb{P}\left(\tilde{S}_{\bullet} \in C\right)$ as assumed in (7). This trading strategy $\phi$ therefore solves (4).

Proof of Theorem 1: Lemma 4 implies Theorem 1 if we let $C_{T}:=\operatorname{pr}_{T}(C)$ with $C$ given by Lemma 4 . Then the trading strategy $\phi$ given by Lemma 4 replicates $1_{C_{T}}\left(\tilde{S}_{T}\right)=1_{C}\left(\tilde{S}_{\bullet}\right)$ and solves $(4)$.

\section{$5 \quad$ Further Results}

We prove in this section the theorems 2 and 3.

Lemma 5: Let a function $f: \mathbb{R}^{2} \mapsto \mathbb{R}$ and a measure $\mu$ on $(\mathbb{R}, \mathcal{B})$ be given, such that $f$ and $\mu$ fulfill the following hypotheses:

(i) $f$ is strictly positive

(ii) $\left\{\begin{array}{l}\text { For all } y \in \mathbb{R} \text { the function } x \mapsto f(x, y) \text { is strictly unimodal; i.e. } \\ f(., y) \text { possesses one and only one local extreme point } x_{y} \text { which is } \\ \text { a global maximizer and } y<y^{\prime} \Rightarrow x_{y}<x_{y^{\prime}}\end{array}\right.$

(iii) $\lim _{x \rightarrow \pm \infty} f(x, y)=0$ for all $y \in \mathbb{R}$ 
(iv) $\mu$ is absolutely continuous with respect to Lebesgue measure and

$$
\int_{-\infty}^{+\infty} f(x, y) d \mu(y)=1
$$

For $A \in \mathcal{B}$ we denote by $f_{A}: \mathbb{R} \mapsto \mathbb{R}$ the function

$$
f_{A}(x):=\int_{A} f(x, y) d \mu(y)
$$

The following conclusions hold:

$$
\left\{\begin{array}{l}
\text { If } A=(-\infty, \hat{a}) \text { for some } \hat{a} \in \mathbb{R}, \text { then } f_{A} \text { is } \\
\text { strictly monotone decreasing, } \lim _{x \rightarrow-\infty} f_{A}(x)=1 \text { and } \\
\lim _{x \mapsto+\infty} f_{A}(x)=0 .
\end{array}\right.
$$

$$
\left\{\begin{array}{l}
\text { If } A=(\hat{a},+\infty) \text { for some } \hat{a} \in \mathbb{R} \text {, then } f_{A} \text { is strictly mono- } \\
\text { tone increasing, } \lim _{x \mapsto-\infty} f_{A}(x)=0 \text { and } \lim _{x \mapsto+\infty} f_{A}(x)= \\
1 .
\end{array}\right.
$$

$$
\left\{\begin{array}{l}
\text { If } A \text { is a bounded subset of } \mathbb{R} \text { and } \mu(A)>0, \text { then } f_{A}(x)>0 \\
\text { for any } x \in \mathbb{R} \text { and } \lim _{x \rightarrow \pm \infty} f_{A}(x)=0 .
\end{array}\right.
$$

Remark: (ii) implies that $f(., y)$ is strictly monotone increasing on $\left(-\infty, x_{y}\right]$ and strictly monotone decreasing on $\left[x_{y},+\infty\right)$.

Proof: We show first:

The measure $\mu$ does not vanish on sets of the form $(-\infty, \hat{a})\}$

We prove this fact by contradiction. Suppose that $\mu(-\infty, \hat{a})=0$ for some $\hat{a} \in \mathbb{R}$. Then for all $x \in \mathbb{R}$

$$
\int_{\hat{a}}^{\infty} f(x, y) d \mu(y)=\int_{-\infty}^{+\infty} f(x, y) d \mu(y)=1 .
$$

For $y>\hat{a}$ we get by hypothesis (ii) (see the preceding Remark) that $x_{y}>x_{\hat{a}}$ and thus that $f(., y)$ is strictly monotone increasing on $\left(-\infty, x_{\hat{a}}\right)$ for $y \geq \hat{a}$. But then we must also have that $x \mapsto \int_{\hat{a}}^{\infty} f(x, y) d \mu(y)$ is strictly monotone increasing on $(-\infty, \hat{a})$ which contradicts $(23)$. So we have proved that $\mu$ does not vanish on sets of the form $(-\infty, \hat{a})$. An analogous argument shows that also the measure of sets of the form $(\hat{a}, \infty)$ is strictly positive and thus $(22)$ is proved. 
As another important fact we note that

$$
f_{(-\infty, \hat{a}]}(x)=1-f_{[\hat{a}, \infty)}(x) .
$$

This fact follows directly from (iv).

We prove now (I).

To prove (I) we show first that for $\hat{a} \in \mathbb{R}$ fixed the function

$$
x \mapsto f_{(-\infty, \hat{a}]}(x)=\int_{(-\infty, \hat{a}]} f(x, y) d \mu(y)
$$

is strictly monotone decreasing on each of the intervals $\left[x_{\hat{a}}, \infty\right),\left(-\infty, x_{\hat{a}}\right]$. That $f_{(-\infty, \hat{a}]}(x)$ is strictly monotone decreasing on $\left[x_{\hat{a}}, \infty\right)$ can be seen as follows:

Since by hypothesis (ii) we have that for $y<\hat{a}$ the unique local maximizer $x_{y}$ of $f(., y)$ is smaller than $x_{\hat{a}}$; i.e. $x_{y}<x_{\hat{a}}$ we see that for $y \leq \hat{a}$ the functions $f(., y)$ are strictly monotone decreasing on $\left[x_{\hat{a}}, \infty\right)$. Therefore and since by (22) the measure $\mu$ does not vanish on sets of the form $(-\infty, \hat{a}]$ we get that also the function $f_{(-\infty, \hat{a}]}=\int_{-\infty}^{\hat{a}} f(., y) d \mu(y)$ is strictly monotone decreasing on $\left[x_{\hat{a}}, \infty\right)$.

An analogous argument shows now that $f_{[\hat{a}, \infty)}(x)$ is strictly monotone increasing on $\left(-\infty, x_{\hat{a}}\right]$ and since by $(24)$ we have $f_{(-\infty, \hat{a}]}=1-f_{[\hat{a}, \infty)}$ we see that $f_{(-\infty, \hat{a}]}$ is also strictly monotone decreasing on $\left(-\infty, x_{\hat{a}}\right]$.

We show next that $\lim _{x \rightarrow \infty} f_{(-\infty, \hat{a}]}(x)=0$ :

We know by hypothesis (iv) that $f_{(-\infty, \hat{a}]}(x) \leq 1$ and by hypothesis (ii) that

$\ldots x_{n}>\ldots>x_{2}>x_{1}>x_{\hat{a}}$ and $y \in(-\infty, \hat{a}]$ implies that

$\ldots>f\left(x_{1}, y\right)>f\left(x_{2}, y\right)>\ldots>f\left(x_{n}, y\right)>\ldots$

We choose now an arbitrary monotone increasing sequence $\left(x_{n}\right)_{n \in \mathbb{N}}, x_{n} \in \mathbb{R}$ with $x_{1}>x_{\hat{a}}$ and $\lim _{n \rightarrow \infty} x_{n}=\infty$. Then by hypothesis (iii) and (25) above the sequence $\left(f\left(x_{n}, y\right)\right)_{n \in \mathbb{N}}$ decreases for each $y \in(-\infty, \hat{a}]$ monotone to 0 . Since $\int_{-\infty}^{\hat{a}} f\left(x_{1}, y\right) d \mu(y) \leq 1$ and $f\left(x_{1}, y\right)>f\left(x_{n}, y\right) \geq 0$ we get by dominated convergence that the sequence $\left(f_{(-\infty, \hat{a})}\left(x_{n}\right):=\int_{-\infty}^{\hat{a}} f\left(x_{n}, y\right) d \mu(y)\right)_{n \in \mathbb{N}}$ tends to 0 . Thus we have proved that $\lim _{x \rightarrow \infty} f_{(-\infty, \hat{a}]}(x)=0$.

Analogous we can show that $\lim _{x \rightarrow-\infty} f_{(\hat{a}, \infty)}(x)=0$. And by (24) we get from this that $\lim _{x \rightarrow-\infty} f_{(-\infty, \hat{a}]}(x)=1$.

Thus (I) has been proved.

Since by (24) we have $f_{[\hat{a}, \infty)}=1-f_{(-\infty, \hat{a})}$ we obtain (II) from (I). 
We finally prove (III).

Suppose that $A$ is bounded and $\mu(A)>0$. Then $f_{A}(x)=\int_{A} f(x, y) d \mu(y)>$ 0 , since $f(x, y)>0$ for all $x, y \in \mathbb{R}$ by hypothesis (i). Let now $\hat{a}, b \in \mathbb{R}$ be such that $A \subseteq[\hat{a}, b]$. Then

$$
0 \leq f_{A}(x) \leq f_{[\hat{a}, b]}(x)=1-f_{(-\infty, \hat{a})}-f_{(b, \infty)}
$$

Since $\lim _{x \mapsto-\infty} f_{(-\infty, \hat{a})}=1$ and $\lim _{x \mapsto \infty} f_{(b, \infty)}=1$ we obtain from (26) that $\lim _{x \rightarrow \pm \infty} f_{A}=0$.

Thus the Lemma has been proved.

Lemma 6: There exists a version of the conditional Probability $\mathbb{P}\left(\ln \left(\tilde{S}_{T}\right)+\right.$ $\left.\frac{T}{2} \in D \mid \ln \left(S_{T}\right)+\frac{T}{2}=y\right)=: \hat{Q}_{T}^{y}(D)$ such that $\hat{Q}_{T}^{y}$ is absolutely continuous with respect to $R_{T}$ and

$$
\frac{d\left(\hat{Q}_{T}^{y}\right)}{d\left(\hat{R}_{T}\right)}=\frac{e^{-\frac{(x-\rho y)^{2}}{2\left(1-\rho^{2}\right) T}+\frac{z^{2}}{2 T}}}{\sqrt{1-\rho^{2}}}
$$

Proof: The result is an immediate consequence of (15) if we consider a proper sequence of sets $\left(A_{T}^{n}\right)_{n \in \mathbb{N}}$ which converges (with respect to the Hausdorff metric) to $y$.

Lemma 7: Let $\rho>0$. Let $f: \mathbb{R}^{2} \mapsto \mathbb{R}$ be defined by

$$
f(x, y)=\frac{e^{-\frac{(x-\rho y)^{2}}{2\left(1-\rho^{2}\right) T}+\frac{x^{2}}{2 T}}}{\sqrt{1-\rho^{2}}}
$$

and let $\mu(y)=\frac{1}{\sqrt{2 \pi T}} e^{-\frac{y^{2}}{2 T}} d \lambda(y)$. Then $f$ and $\mu$ fulffll the hypotheses of Lemma 5 .

Proof: Hypothesis (i) of Lemma 5 follows from the strict positivity of the exponential function. Since we assumed $\rho>0$ an easy calculation shows that (ii) holds with $x_{y}=\frac{y}{\rho}$. It is also not difficult to see that (iii) holds if we keep in mind that we assumed $\rho>0$. So it remains to prove that hypothesis (iv) is fulfilled. That $\mu$ is absolutely continuous with respect to Lebesgue measure follows from its definition. To show that $\int_{-\infty}^{+\infty} f(x, y) d \mu(y)=1$ we note that $\hat{Q}_{T}^{(-\infty,+\infty)}=\hat{R}_{T}$ (which follows easily from the definition of $Q, R, \hat{Q}$ and $\hat{R}$ ). So the integral can be calculated as follows:

$$
\int_{-\infty}^{+\infty} f(x, y) d \mu(y)=\frac{\int_{-\infty}^{+\infty} \frac{e^{-\frac{(x-p y)^{2}}{2\left(1-p^{2}\right)}+\frac{x^{2}}{2 T}}}{\sqrt{1-p^{2}}} \frac{1}{\sqrt{2 \pi T}} e^{-\frac{y^{2}}{2 T}} d \lambda(y)}{1}=
$$




$$
\begin{gathered}
=\frac{\int_{-\infty}^{+\infty} \frac{e^{-\frac{(x-\rho y)^{2}}{2\left(1-\rho^{2}\right) T}+\frac{x^{2}}{2 T}}}{\sqrt{1-\rho^{2}}} \frac{1}{\sqrt{2 \pi T}} e^{-\frac{y^{2}}{2 T}} d \lambda(y)}{\int_{-\infty}^{+\infty} \frac{1}{\sqrt{2 \pi T}} e^{-\frac{y^{2}}{2 T}} d \lambda(y)}= \\
\frac{d\left(\hat{Q}_{T}^{(-\infty,+\infty)}\right)}{d\left(\hat{R}_{T}\right)}=\frac{d\left(\hat{R}_{T}\right)}{d\left(\hat{R}_{T}\right)}=1
\end{gathered}
$$

Proof of Theorem 2: We will only prove the Theorem in case 1; i.e. in the case $A_{T}:=(0, a]$. We show first that there exists $\bar{c} \in \mathbb{R}$ such that $C_{T}:=(0, \bar{c}]$ fulfills condition (11). By Proposition 1 and equation (18) it is sufficient for this to show that $\frac{d\left(Q_{T}^{[0, a]}\right)}{d\left(R_{T}\right)}$ is monotone decreasing, which is clearly equivalent to the fact that $\frac{d\left(\hat{Q}_{T}^{\left(-\infty, \ln (\hat{\alpha})+\frac{T}{2}\right)}\right)}{d\left(\hat{R}_{T}\right)}$ is monotone decreasing. But this is a consequence of Lernma 5 and Lemma 7 , since if we let $f(.,$.$) and \mu$ be defined as in Lemma 6 , then

$$
\frac{d\left(\hat{Q}_{T}^{\left(-\infty, \ln (a)+\frac{T}{2}\right]}\right)}{d\left(\hat{R}_{T}\right)}(x)=\int_{-\infty}^{\ln (a)+\frac{T}{2}} f(x, y) d \mu(y)
$$

which is by conclusion (I) of Lemma 5 (with $\hat{a}:=\ln (a)+\frac{T}{2}$ ) a monotone decreasing function. So we proved that $C_{T}$ is of the form $(0, \bar{c}]$ and thus it remains only to show that $\bar{c}=e^{F_{T}^{-1}\left(v_{0}\right)-\frac{T}{2}}$. But since by Lemma 4 and Theorem 1 the set $\operatorname{pr}_{T}^{-1}((0, \bar{c}])$ fulfills (7) we have by (2)

$$
F_{T}(\ln (\bar{c}))+\frac{T}{2}=\mathbb{P}\left(\tilde{S}_{T} \in(0, \bar{c}]\right)=\mathbb{P}\left(\tilde{S}_{\bullet} \in p r_{T}^{-1}((0, \bar{c}])\right)=v_{0}
$$

Proof of Theorem 3: We know by Theorems 1 and 2 that the set $C_{T}$ which determines our optimal hedge equals a.s. $p r_{T}^{-1}((0, c(\varepsilon)])$, with $c(\varepsilon)$ a real number depending on $\varepsilon$. Since $S_{T}$ and $\tilde{S}_{T}$ possess the same (marginal) distribution, our assumptions imply that

$$
R_{T}((0, c(\varepsilon)])=v_{0}=\mathbb{E}_{\mathbb{P}}(H)+\varepsilon=R_{T}((0, a])+\varepsilon .
$$

Since $R_{T}$ is absolutely continuous with respect to Lebesgue measure and $\frac{d R_{T}}{d \lambda}$ is bounded, we obtain from $(27)$ that $c(\varepsilon)>a+\varepsilon k$ for a suitable constant $k>0$. The hedging error $\operatorname{Err}(\rho, \varepsilon, a, T)$ is now given by

$$
\begin{gathered}
\operatorname{Err}(\rho, \varepsilon, a, T)=\mathbb{P}\left(V_{T}^{\phi}<H\right)=1-\mathbb{P}\left(V_{T}^{\phi} \geq 1 \mid S_{T} \in(0, a]\right) \\
=1-\mathbb{P}\left(\tilde{S}_{\bullet} \in C_{\phi} \mid S_{T} \in(0, a]\right)=1-\mathbb{P}\left(\tilde{S}_{T} \in(0, c(\varepsilon)] \mid S_{T} \in(0, a]\right)
\end{gathered}
$$




$$
\begin{aligned}
& \leq 1-\mathbb{P}\left(\tilde{S}_{T} \in(0, a+\varepsilon k] \mid S_{T} \in(0, a]\right)=\mathbb{P}\left(\tilde{S}_{T} \in[a+\varepsilon k, \infty) \mid S_{T} \in(0, a]\right) \\
& =\mathbb{P}\left(\ln \left(\tilde{S}_{T}\right)+\frac{T}{2} \in\left[\ln (a+\varepsilon k)+\frac{T}{2}, \infty\right) \mid \ln \left(S_{T}\right)+\frac{T}{2} \in\left(-\infty, \ln (a)+\frac{T}{2}\right]\right) \\
& =\hat{Q}_{T}^{\left(-\infty, \ln (a)+\frac{T}{2}\right]}\left(\left[\ln (a+\varepsilon k)+\frac{T}{2},+\infty\right)\right) \\
& \leq \hat{Q}_{T}^{\left(-\infty, \ln (a)+\frac{T}{2}\right]}\left(\left[\ln (a)+\frac{T}{2}+\varepsilon(\ln (a+k)-\ln (a)), \infty\right)\right) \\
& \leq \hat{Q}_{T}^{\ln (a)+\frac{T}{2}}\left(\left[\ln (a)+\frac{T}{2}+\varepsilon(\ln (a+k)-\ln (a)), \infty\right)\right) \\
& =\int_{\ln (a)+\frac{T}{2}+\varepsilon(\ln (a+k)-\ln (a))}^{\frac{d \hat{Q}_{T} \ln (a)+\frac{T}{2}}{d \hat{R}_{T}}} \frac{d \hat{R}_{T}}{d \lambda} d \lambda \\
& =\int_{\ln (a)+\frac{T}{2}+\varepsilon(\ln (a+k)-\ln (a))}^{+\infty} \frac{e^{-\frac{\left(x-\rho\left(\ln (a)+\frac{T}{2}\right)^{2}\right.}{2\left(2-p^{2}\right) T}}}{\sqrt{1-\rho^{2}} \sqrt{2 \pi T}} d \lambda(x) \\
& \leq \frac{e^{-\frac{\left(\ln (a)+\frac{T}{2}+\varepsilon(\ln (a+k)-\ln (a))-\rho\left(\ln (a)+\frac{\pi}{2}\right)^{2}\right.}{2\left(1-\rho^{2}\right) T}}}{\sqrt{1-\rho^{2}} \sqrt{2 \pi T}} \leq \frac{e^{-\frac{(\varepsilon \ln (a+k)-\ln (a))^{2}}{2\left(1-\rho^{2}\right) T}}}{\sqrt{1-\rho^{2} \sqrt{2 \pi T}}}
\end{aligned}
$$

Here the $3^{\text {rd }}$ equality follows by Theorem 1 , the $4^{\text {th }}$ by Theorem 1 and Theorem 2 ; the $1^{s t}$ inequality follows since by $(27) c(\varepsilon)>a+\varepsilon k$. The $7^{\text {th }}$ equality follows from (13), the $2^{\text {nd }}$ inequality follows by concavity of the logarithm and the $3^{\text {rd }}$ inequality by the definition of $\hat{Q}_{T}^{y}$ in Lemma 6 and an application of Lemma 7 and Lemma 5. The last equality follows by the definition of $\hat{Q}_{T}^{y}$ and the $4^{\text {th }}$ inequality follows easily from the well known fact that for $y \geq 0, e^{\frac{y}{2}} \geq \int_{y}^{\infty} e^{-\frac{t}{2}} d t$. Finally, since $\varepsilon, k>0$ an easy calculation shows that the last expression tends for $\rho \rightarrow 1$ faster to 0 than any polynomial in $1-\rho$.

\section{Appendix}

We reinvestigate theorems 1-3 in a slightly more general setting (MGS). We allow drift terms $\mu$ and $\tilde{\mu}$ in our model: 


$$
\text { (MGS) }\left\{\begin{array}{l}
\text { Let } W \text { and } \tilde{W} \text { be correlated Brownian motions with correlation } \\
\rho \in(-1,+1) \backslash\{0\} . \text { Let } S \text { and } \tilde{S} \text { be assets with price processes } \\
\text { given by: } \\
\qquad \frac{d S_{t}}{S_{t}}=d W_{t}+\mu, S(0)=1 \text { and } \\
\frac{d \tilde{S}_{t}}{\tilde{S}_{t}}=d \tilde{W}_{t}+\tilde{\mu}, \tilde{S}(0)=1
\end{array}\right.
$$

$\rho \in(-1,+1) \backslash\{0\}$. Let $S$ and $\tilde{S}$ be assets with price processes

and let $B$ be a bond with interest rate $e^{\bar{\mu}}-1$.

Note that $\tilde{S}$ and $B$ form a complete financial market and that in analogy with (2) we obtain:

$$
\left(\begin{array}{l}
\ln \left(S_{T}\right)+\frac{T}{2}-\mu T \\
\ln \left(\tilde{S}_{T}\right)+\frac{T}{2}-\tilde{\mu} T
\end{array}\right) \sim N\left(\left(\begin{array}{l}
0 \\
0
\end{array}\right)\left(\begin{array}{cc}
T & \rho T \\
\rho T & T
\end{array}\right)\right) .
$$

Since all the results derived in this paper are derived from (2) and the fact that the market formed by $\tilde{S}$ and $B$ is complete, Theorems $1-3$ remain essentially true:

Theorem 1': Theorems 1 and 3 remain true under MGS.

Theorem 2': Under MGS Theorem 2 remains true if we denote by $F_{T}$ the distribution function of $N(\mu T, T)$ instead of $N(0, T)$.

\section{References}

[1] M. Amram, N. Kulatilaka (1999): Real Options: Harvard Business School Press

[2] M. Amram, N. Kulatilaka (1999): Disciplined Decisions. Harvard Business Review, January-February, 95-104

[3] H. Foellmer, P. Leukert (1999): Quantile Hedging. Finance and Stochastics 3(1999) 3, 251-273

[4] H. Foellmer, P. Leukert (2000): Efficient Hedging: Costs versus Shortfall Risk. Finance and Stochastics 4(2000) 2, 117-146

[5] E. L. Lehmann (1994): Testing Statistical Hypotheses 2nd. ed.: Chapman \& Hall 
[6] T. A. Luehrman (1998): Strategy as a Portfolio of Real Options. Harvard Business Review, September-October

[7] J. M. Steele (2001): Stochastic Calculus and Financial Applications: Springer Verlag New York, Berlin, Heidelberg

Authors Address:

Heinz Weisshaupt

EURANDOM, P.O. Box 513

$5600 \mathrm{MB}$ Eindhoven

The Netherlands 